\title{
Communications
}

\section{Société Suisse de Neurologie (SSN)}

\section{Comité de la SSN dès le 12 juin 2009}

Président:

Prof. Dr C. Bassetti, Lugano

Président sortant:

Dr Max Wiederkehr, Lucerne

Vice-président:

Prof. Dr Pierre Burkhard, Genève

Secrétaire:

Prof. Dr Philipp Lyrer, Bâle

\section{Trésorier:}

Dr U. Venetz, Viège

Assesseurs:

Dr M. Corboz, Payerne

Prof. Renaud Du Pasquier, Lausanne

Prof. René Müri, Berne

Office SSN:

Dr Catherine Perrin

Directrice

15, avenue des Planches

CH-1820 Montreux

info@swissneuro.ch

\section{Schweizerische Gesellschaft \\ der Vertrauens- und Versicherungs- ärzte (SGV) / Société Suisse \\ des médecins-conseils et \\ des médecins d'assurance (SSMC)}

\section{Vorstand}

Präsident:

Dr. med. Jürg Zollikofer, Ossingen juerg.zollikofer@hin.ch

Vize-Präsident:

Dr. med. Daniel Loustalot

Quästor:

Dr. med. Beat Gründler

Leiter Weiterbildungskommission:

Dr. med. Fredi Bacchetto

Beisitzer:

Dr. med. Didier Grob

Dr. med. Géza Kanabé

Dr. med. Valentin Rehli

Dr. med. Paul Vogt

Dr. med. Frédérique Zihlmann

Geschäftsführer / Sekretär:

Markus Bonelli

Geschäftsstelle:

c/o MBC Markus Bonelli Consulting

Wülflingerstrasse 59

CH-8400 Winterthur

markus.bonelli@bonelli.ch

info@vertrauensaerzte.ch

info@medecins-conseils.ch

info@medici-fiduciari.ch

\section{$A B C$ Arbeit und Behinderung}

Die IVB Behindertenselbsthilfe beider Basel engagiert sich für die Integration von Menschen mit einer Behinderung. Im Rahmen dieser Tätigkeit hat sie für Arbeitgeber, Ärzte, Sozialberatende und Betroffene den Ratgeber «Arbeit und Behinderung» publiziert. Das

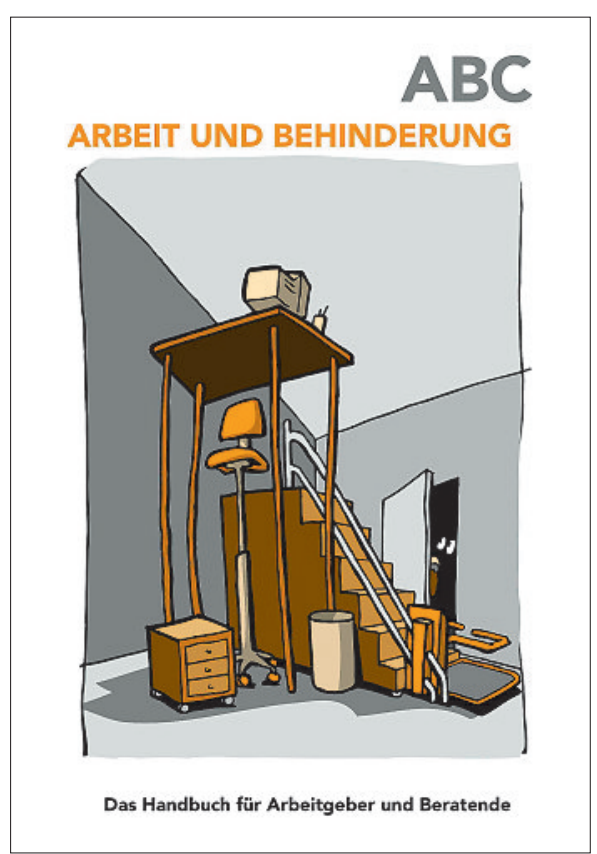

Nachschlagewerk für die gesamte Schweiz informiert über die komplexe Thematik und beantwortet eine Vielzahl von Fragen. Die Themenschwerpunkte: Problemfelder, Vorurteile, Rückkehr und Eingliederung in die Arbeitswelt, Anreize und Unterstützungsleistungen, angepasster Arbeitsplatz, Beratungsstellen, hindernisfreies Bauen, Invalidenversicherung (allgemeine Fragen und Antworten zur IV und den Neuerungen), Definitionen und Begriffserklärungen $\mathrm{A}-\mathrm{Z}$, Medizinisches A-Z, Behinderung und Recht, Sozialversicherungen und Sozialeinrichtungen A-Z. Zu allen Themen finden sich weiterführende Literaturangaben, Links und Adressen.

Weitere Informationen:ivb@ivb.ch 